\title{
Corona Performance in Wire-cylinder ESP with Particle Loading
}

\author{
Mazen Abdel-Salam ${ }^{1 . a}$, Mohamed Th. El-Mohandes ${ }^{1}$ and Seham Kamal El-deen ${ }^{1}$ \\ ${ }^{1}$ Electrical Engineering Department, Assiut University, Assiut, Egypt
}

\begin{abstract}
This paper is aimed at investigating thoroughly the corona performance in the wire-cylinder electrostatic precipitor (ESP) with loading by suspended particles in the exhaust of a diesel engine. The onset voltage of negative corona on the discharge wire is calculated based on the criterion of self-sustained discharge. The ionized field in the ESP is mathematically modeled for calculating the spatial distribution of the space-charge density due to both the ions and the charged particles as well as the components of the electric field including the applied field and the field due to the space charge. This is in addition to the calculation of the current-voltage characteristics of the ESP with particle loading.
\end{abstract}

\section{Introduction}

The electrostatic precipitator is the most effective device to collect industrial particulates before escaping into the atmosphere to pollute it. This is why the researchers over the years work on modeling the processes inside the electrostatic precipitors(ESPs) including corona discharge seeking improvement of their performance.

An iterative model employed the finite element method for computing electric potential structure for an assumed charge density distribution and the doner cell method was used to compute charge density structure for an assumed electric field distribution. The model can account for particle loading of the ESP and bipolar ionic species resulting from back-ionization at the collecting electrode [1].

The Poisson's equation was solved in wire-duct ESP with particle loading using the finite difference method for a given ion density distribution. The ion charge density at the boundary of the ionization-zone was evaluated empirically and used to determine the ion-density spatial distribution. The effect of assumed uniform particle charge density on the current-voltage characteristic of the ESP was investigated, where the corona onset voltage increases with the increase of the particle charge density. Subsequently, the ion current decreases with the increase of particle charge density at the same applied voltage [2]. In wire-duct ESP, the effect of particle loading and applied voltage on the mean radius of the ionization zone around the discharge wires was investigated[3].

In wire-duct ESP, the finite element method was used to determine the electric field and current density distributions based on Kaptzov's assumption which was considered valid for each corona electrode[4]. The electric field on the corona electrode surfaces is equal to the corona onset field, given by Peek's formula[5]. Non-justified simplifying assumptions have been additionally introduced

\footnotetext{
${ }^{\text {a } C o r r e s p o n d i n g ~ a u t h o r: ~ m a z e n 2000 a s @ y a h o o . c o m . ~}$
} 
in the mathematical model: 1) It is supposed that the electric field on the surface of each corona electrode is virtually uninfluenced by the presence of particles. Subsequently, the corona onset voltage is not influenced by the presence of particles. 2) Ionic charge density is also virtually uninfluenced by the presence of particles.

The current-voltage formula for wire-duct ESP was derived where the particle space charge was assumed uniform over the cross-section of the precipitator. This concluded that the onset voltage of corona increases with the particle loading [6].

Simplifying assumptions commonly adopted in the ESP modeling which are justified:

1. Kaptsov' assumption which states that the field at the coronating surface remains constant at the corona onset value as determined by Peek's formula [5] irrespective of the value of the applied voltage.

2. The mobility of ions remains constant as their transit time from the discharge wire to the collecting electrode is so small for the mobility to change with the ions' life time.

Other simplifying assumptions were adopted without justification for the ESP with particle loading:

1) Some investigators $[2,3]$ evaluated empirically the ion charge density at the boundary of the ionization-zone surrounding the discharge wire in order to determine the ion density distribution.

2) Some investigators [4] assumed the electric field on the surface of the discharge wire is virtually uninfluenced by the presence of particles.

3) Some investigators [4] assumed the ion charge density is virtually uninfluenced by the presence of particles.

4) Some investigators [6] assumed uniform particle space charge density over the ESP cross section.

5) With particle loading, some investigators [7, 8] assumed that the charge acquired by particles is related to the field component due to the ion space charge with no justification.

This motivates the authors to investigate thoroughly the corona performance in the wire-cylinder ESP when loaded with suspended particles in the exhaust of diesel engine.

\section{Method of Analysis}

The equations describing the electric field and flow of charges carriers (ions and charged particles) in the ESP are the Poisson's equation for the electric field, the equation for current density and the equation of current continuity[9].

Poisson's equation for the electric field is expressed as:

$$
\nabla . E=\frac{\rho}{\epsilon_{0}}
$$

where $\mathrm{E}$ is the electric field with space charge including corona ions and charged particles, $\rho$ is the total volume charge density of ions and particles and $\epsilon_{\mathrm{o}}$ is permittivity of free space.

The total volume charge density is expressed as:

$$
\rho=\rho_{i}+\rho_{p}
$$

where $\rho_{i}$ is the ion charge density and $\rho_{p}$ is the particle charge density.

The electric field $\mathrm{E}$ is expressed as:

$$
E=E_{f+} E_{s}
$$

where $E_{f}$ is the electric field without space charge i.e.; the field due to the applied voltage and $E_{\mathrm{s}}$ is the field due to space charge of ions and charged particles.

In a wire-cylinder geometry of the precipitator, the space charge affects only the magnitude but not the direction of the electric field, i.e.;

$$
E=\xi E_{f}
$$

where $\xi$ is a scalar which changes over the space between the discharge wire and the collecting cylinder.

From equations (3) and (4), the field $\mathrm{E}_{\mathrm{s}}$ due to space charge is expressed as: 


$$
E_{s}=(\xi-1) E_{f}
$$

The equation of precipitator current density is expressed as:

$$
J=k_{i} \rho_{i} E+k_{p} \rho_{p} E
$$

where $k_{i}$ and $k_{p}$ are the mobility of corona ions and charged particles, respectively.

Equation (6) of the current density is rewritten as:

where the mobility ratio $k$ is expressed as:

$$
J=k_{i} E\left(\rho_{i}+k \rho_{p}\right)
$$

$$
k=\frac{k_{p}}{k_{i}}
$$

The particles are charged by ion-bombardment process, which is the dominant charging mechanism for particles of radii greater than $1 \mu \mathrm{m}[10]$. The particle charge density is expressed as:

$$
\rho_{p}=\epsilon_{o} \lambda E S
$$

where:

$$
\lambda=\frac{2 \epsilon_{r}}{\epsilon_{r}+3}
$$

where $\epsilon_{\mathrm{r}}$ is the relative permittivity of the particles and $\mathrm{S}$ is the particle specific surface.

Assuming the particles are spherical in shape with radius $r_{p}$, so $S$ is expressed as:

$$
S=4 \pi r_{p}^{2} N_{p}
$$

where $\mathrm{N}_{\mathrm{p}}$ is the particles concentration being assumed constant over the cross-section of the particle.

The particle concentration $N_{p}$ is as expressed as follows:

$$
N_{p}=\frac{Z}{\gamma \frac{4}{3} \pi r_{p}^{3}}
$$

where $\mathrm{Z}$ is the particles weight per unit volume and $\gamma$ is the specific gravity of the particles.

With the use of equation (4), the particle charge $\rho_{p}$ density of eq. (9) is expressed as:

$$
\rho_{p}=A \xi E_{f}
$$

where $A=\epsilon_{o} \lambda \mathrm{S}$ is a constant for particles of the same radius and permittivity.

The mobility $k_{p}$ of the particle with charge $\mathrm{q}_{\mathrm{p}}$ is determined by balancing the electric force $\left(=\mathrm{q}_{\mathrm{p}} . \mathrm{E}\right)$ with Stokes drag force $\left(=6 \pi \eta r_{p} k_{p} E\right)$ where $\eta$ is the viscosity of the gas inside the precipitator.

Therefore, the particle mobility $\mathrm{k}_{\mathrm{p}}$ is expressed as:

The particle charge $\mathrm{q}_{\mathrm{p}}$ is expressed as:

$$
k_{p}=\frac{q_{p}}{6 \pi \eta r_{p}}
$$

$$
q_{p}=\frac{\rho_{p}}{N_{p}}
$$

Combination of equations (1), (2), (4) and (13) results in the following differential equation, which defines the spatial distribution of the scalar $\xi$ over the space between the discharge wire and collecting cylinder:

$$
\frac{d \xi}{d r}=\frac{\rho_{i}+A \xi E_{f}}{\epsilon_{0} E_{f}}
$$

The continuity equation of the precipitator current density is expressed as:

$$
\nabla . J=0
$$

Combination of equations (4), (7) and (17) results in the following differential equation:

$$
\frac{d \rho_{i}}{d \xi}=-\left(\frac{\rho_{i}+2 k A \xi E_{f}}{\xi}\right)
$$

Combining of equations (16) and (18) results in the following differential equation which defines the spatial distribution of the ion charge density $\rho_{i}$ over the space between the discharge wire and collecting cylinder:

$$
\frac{d \rho_{i}}{d r}=-\left(\frac{\rho_{i}+2 K A \xi E_{f}}{\xi}\right)\left(\frac{\rho_{i}+A \xi E_{f}}{\epsilon_{o} E_{f}}\right)
$$

The differential equations (16) and (19) are integrated along the radial direction to determine the spatial distribution of $\xi$ and $\rho_{\mathrm{i}}$ over the space between the discharge wire and collecting cylinder. The 
initial conditions for such integrals are (i) $\xi$ at discharge wire is equal to $\mathrm{V}_{\mathrm{o}} / \mathrm{V}$, where $\mathrm{V}_{\mathrm{o}}$ is the corona onset voltage determined as explained elsewhere [11] and V is the applied voltage. (ii) The electric field at the surface of the discharge electrode remains constant at the onset value, which is Kaptsov's assumption.

\section{Results and Discussions}

Figure (1) shows the slight increase of $\mathrm{E}_{\mathrm{s}}$ with particle loading when compared with that for the case without particle loading [11]. The integration of $\mathrm{E}_{\mathrm{s}}$ along the spacing between the discharge wire and collecting cylinder remains very close to zero. Subsequently, the integration of the electric field E over the spacing between the wire and cylinder is almost equal to the applied voltage which satisfies the Dirichlet condition. The onset voltage $V_{o}^{\prime}$ with particle loading exceeds that $\mathrm{V}_{\mathrm{o}}$ without particle loading in agreement with eq. (A-2) of Appendix 1.

Figure (2) shows the spatial distribution of the electric field E over the space from the ionizationzone boundary to the cylinder as predicted by eq. (A-1) of Appendix 1 in comparison with that obtained using the present method of calculation. The deviation in Fig. (2) from the present calculated values is attributed to the fact that eq. (A-1) is based on assuming the same particle charge density over the cross section of the ESP.

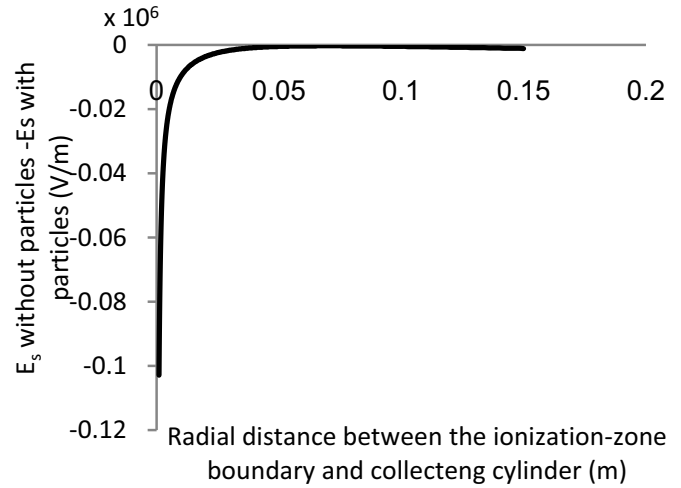

Figure 1: Excess of Es with particle loading over that without particle loading between the ionization-zone boundary and collecting cylinder. $\mathrm{r}_{\mathrm{o}}=1 \mathrm{~mm}, \mathrm{R}=15 \mathrm{~cm}$, $\mathrm{V}_{\mathrm{o}}{ }^{\prime}=30.659 \mathrm{kV}$ and $\mathrm{V}=60 \mathrm{kV}$.

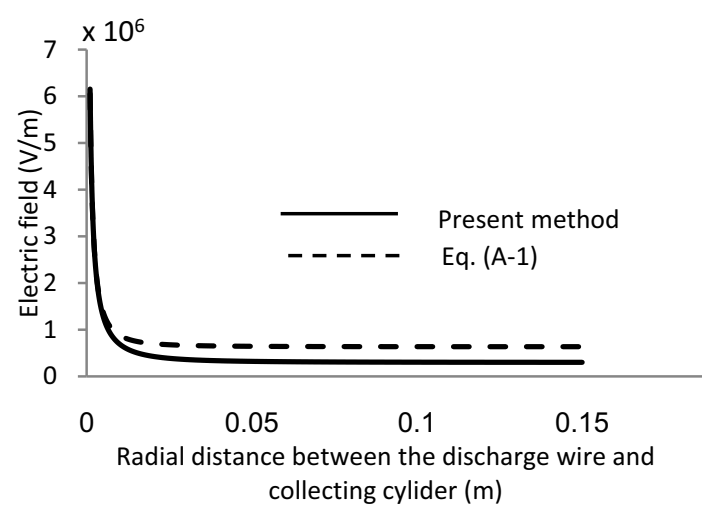

Figure 2: Calculated spatial distribution of the electric field $\mathrm{E}$ between the discharge wire and collecting cylinder compared with that obtained by eq. (A-1) for the ESP with particle loading. $r_{o}=1$ $\mathrm{mm}, \mathrm{R}=15 \mathrm{~cm}, \mathrm{~V}_{\mathrm{o}}{ }^{\prime}=30.659 \mathrm{kV}$ and $\mathrm{V}=60 \mathrm{kV}$.

Figure (3) shows the spatial distribution of the ion charge density over the spacing between the wire and cylinder with and without particle loading [11]. The spatial distribution of the $\rho_{\mathrm{p}}$ in Fig. (3) follows that of the electric field which charges the particle. This does not support one of the assumptions proposed before [4] which consider no effect of particle loading on the ion charge density. With particle loading, it is worthy to notice in Fig. (3) that (i) $\rho_{\text {p }}$ assumes values almost one order smaller in magnitude than those of $\rho_{\mathrm{i}}$ with particle loading in agreement with previous findings [1] and (ii) the sum $\rho_{\mathrm{p}}+\rho_{\mathrm{i}}$ with particle loading is almost equal to $\rho_{\mathrm{i}}$ without particle loading over the spacing between wire and cylinder. This is because the particles are charged at the expense of ions.

Figure (4) shows how the corona current increases with the applied voltage with and without particle loading. The figure shows a slight increase of the corona current value without particle loading over that with particle loading at the same voltage.

The decrease of the electric field within the ionization zone due to charged-particles field-shielding results in an increase of the onset voltage $V_{o}^{\prime}$ with the increase of particle radius $r_{p}$ as given in Table (1). The corona I-V characteristics of the ESP at varying $r_{p}$ for the same particle density $N_{p}$ showed an increase of the corona current with the decrease of $r_{p}$ due to decrease of $V_{o}$. Such increase of the corona current reaches up to $10 \%$ at applied voltage of about three times the corona onset voltage $\mathrm{V}_{\mathrm{o}}$ '. 
The decrease of the electric field within the ionization zone due to increase of $\mathrm{N}_{\mathrm{p}}$ because of the above-mentioned field-shielding results in increase of the corona onset voltage $\mathrm{V}_{\mathrm{o}}{ }^{\prime}$ as given in Table (2). The corona I-V characteristic of the ESP at varying particle concentration $\mathrm{N}_{\mathrm{p}}$ for the same particle radius $r_{p}$ showed a decrease of the corona current with the increase of the $\mathrm{N}_{\mathrm{p}}$ because of the increase of $\mathrm{V}_{\mathrm{o}}^{\prime}$.
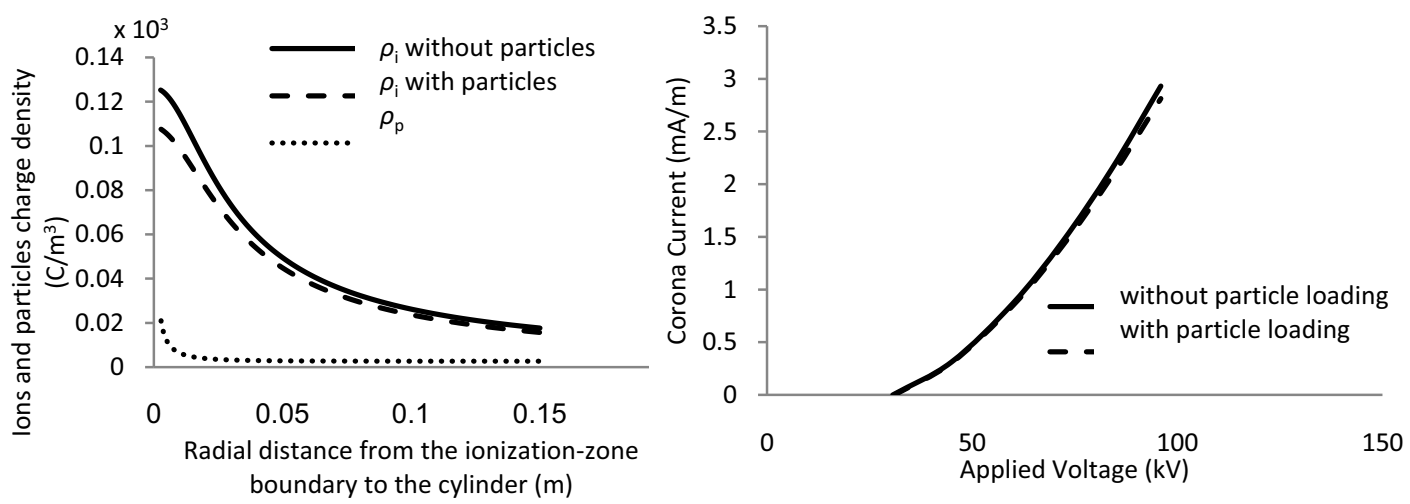

Figure 3: Calculated spatial distribution of the ion and particle charge density values between the discharge wire and collecting cylinder. $\mathrm{r}_{\mathrm{o}}=$ $1 \mathrm{~mm}, \mathrm{R}=15 \mathrm{~cm}, \mathrm{~V}_{\mathrm{o}}=30.659 \mathrm{kV}$ and $\mathrm{V}_{\mathrm{o}}{ }^{\prime}=$ $31.0271 \mathrm{kV}$ and $\mathrm{V}=60 \mathrm{kV}$.

Table 1: Calculated increase of the corona onset voltage $\mathrm{V}_{\mathrm{o}}^{\prime}$ with the increase of particle radius $r_{\mathrm{p}}$ at the same particle concentration $\mathrm{N}_{\mathrm{p}}\left(=0.2 * 0.094 * 10^{9}\right)$.

\begin{tabular}{|c|c|c|c|}
\hline $\mathrm{r}_{\mathrm{p}}(\mu \mathrm{m})$ & 10 & 30 & 50 \\
\hline $\mathrm{V}_{\mathrm{o}}(\mathrm{kV})$ & 30.6898 & 31.57891 & 33.72505 \\
\hline
\end{tabular}

Table 2: Calculated increase of the corona onset voltage $\mathrm{V}_{\mathrm{o}}^{\prime}$ with the increase of particle concentration $\mathrm{N}_{\mathrm{p}}$ at the same particle radius $r_{p}(=30 \mu \mathrm{m})$.

\begin{tabular}{|c|c|c|c|}
\hline $\mathrm{r}_{\mathrm{p}}(\mu \mathrm{m})$ & 10 & 30 & 50 \\
\hline $\mathrm{V}_{\mathrm{o}}(\mathrm{kV})$ & 30.6898 & 31.57891 & 33.72505 \\
\hline
\end{tabular}

\section{Conclusions}

1. The ionized field in the ESP is mathematically modeled for calculating the spatial distribution of space charge density due to both the ions and charged particles as well as the components of electric field including the applied field and the field due to space charge.

2. The calculated spatial distribution of space charge density due to charged particles between the discharge wire and cylinder of the ESP with particle loading is one order higher than that due to ions.

3. The current-voltage characteristics are calculated for the ESP with particle loading at varying particle radius for the same particle concentration. The smaller the particle radius, the higher is the corona current at the same applied voltage.

4. The current-voltage characteristics are calculated for the ESP with particle loading at varying particle concentration for the same particle radius. The higher the particle concentration, the smaller is the corona current at the same applied voltage.

5. The calculated onset voltage of corona on the discharge wire of the ESP with particle loading increases with the increase of either the particle radius or the particle concentration.

\section{Acknowledgment}

This paper is extracted from a research project sponsored through joint innovative projects fund STDF (Egypt) - IRD (France), project STDF ID-5457. The authors acknowledge such sponsorship. 


\section{References}

1. P. L. Levin and J. F. Hoburg, "Donor cell-finite element descriptions of wire-duct precipitator fields, charges, and efficiencies," IEEE Trans. Ind. Appl., IA-26, 4,(1990).

2. M. R. Talaie, M. Taheri, and J. Fathikaljahi, "A new method to evaluate the voltage-current characteristics applicable for a single-stage electrostatic precipitator," J. Electrostat., 53, 3(2001).

3. M. R. Talaie, "Mathematical modeling of wire-duct single-stage electrostatic precipitators.," J. Hazard. Mater., 124, 1-3, (2005).

4. S. Cristina and M. Feliziani, "Calculation of Ionized Fields in DC Electrostatic Precipitators in the Presence of Dust and Electric Wind", IEEE Transactions on Industry Applications, IA-77, 1446-1451(1995).

5. F.W. Peek, "Dielectric Phenomena in High-Voltage Engineering", New Work: McGraw-Hill, (1929).

6. G. Cooperman, “A New Current-Voltage Relation for Duct Precipitators Valid for Low and High Current Densities," IEEE Trans. Ind. Appl., IA-17, 2(1981).

7. A. D. Moore, "Electrostatics and its Applications", J. Wiley \& Sons, New York, USA, 1973.

8. S. Abdel-Satar and H. Singer, "Electrical conditionsin -wire-duct electrostatic," Journal of Electrostatics, 26, 1-20(1991).

9. M. Abdel-Salam, L. Fouad, S. El-Hazek and M. Abu-Hussin, "Wire-Tube Electrostatic Precipitator Cement Factories", Conference record - Industry Applications Socity Annual meeting, San Fransisco, USA, 1129-1135(1982).

10. M. Abdel-Salam and Z. Al-Hamouz, "A New Finite Element Solution of Ionized Field in Coaxial Cylindrical Geometry", J. Phys. D: Applied Physics, 25, 1551-1555(1992).

11. M. Abdel-Salam, M. El-Mohandes and S, Kamal El-deen, "Corona Performance in WireCylinder ESP withot Particle loading", Asia Conference on Power and Electrical Engineering (ACPEE 2016), Bangkok, Thailand, March 20-22, 2016.

\section{Appendices}

\section{Appendix 1: Analytical expressions defining wire-cylinder ESP performance with particle loading:}

\section{Spatial distribution of electric field $E$ :}

It has been reported [7] that the spatial distribution of the electric field with space charge E (r) in wirecylinder ESP is expressed as:

$$
E(r)=\sqrt{\left[\left(\frac{r_{0}}{R}\right)^{2}\left(E_{0}^{2}-\frac{I}{2 \pi \epsilon_{0} K_{i}}\right)+\frac{I}{4 \pi \epsilon_{0} K_{i}(\lambda S r)^{2}}\right] e^{2 \lambda S r}-\frac{I}{4 \pi \epsilon_{0} K_{i}}\left[\frac{2}{\lambda S r}+\frac{1}{(\lambda S r)^{2}}\right]}
$$

\section{Onset voltage of corona:}

It has been reported $[6,7]$ that the corona onset voltage in wire-cylinder ESP increases with the presence of dust as expressed by:

$$
\mathrm{V}_{\mathrm{o}}^{\prime}=\mathrm{V}_{\mathrm{o}}+\frac{\rho_{\mathrm{p}}}{4 \epsilon_{\mathrm{o}}} \mathrm{R}^{2}
$$

where $V_{o}$ and $\mathrm{V}_{\mathrm{o}}^{\prime}$ are the corona onset voltage values with and without particle loading.

The expressions (A-1) and (A-2) are based on assuming both particle $\rho_{\mathrm{p}}$ and ion $\rho_{\mathrm{i}}$ space charge density values are independent of position over the ESP cross-section which is not justified. This makes the formulation of these expressions are questionable $[6,7]$. 